\title{
Strong Convergence Theorems for Nonexpansive Nonself-mappings in Banach Spaces
}

\author{
Gang-Eun Kim and Wataru Takahashi \\ Key words and Phrases: Nonexpansive mapping, sunny retraction, fixed point, weak \\ inwardness condition, strong convergence theorem.
}

\section{Introduction}

Let $E$ be a Banach space and let $C$ be a nonempty closed convex subset of $E$. A mapping $T$ from $C$ into $E$ is called nonexpansive if $\|T x-T y\| \leq\|x-y\|$ for all $x, y \in C$. For a given $u \in C$ and each $t \in(0,1)$, we define a contraction $T_{t}: C \rightarrow E$ by

$$
T_{t} x=t T x+(1-t) u \quad \text { for all } x \in C \text {. }
$$

If $T(C) \subset C$, then $T_{t}(C) \subset C$. Thus, by Banach's contraction principle, there exists a unique fixed point $x_{t}$ of $T_{t}$ in $C$, that is, we have

$$
x_{t}=t T x_{t}+(1-t) u
$$

A question naturally arises to whether $\left\{x_{t}\right\}$ converges strongly as $t \rightarrow 1$ to a fixed point of $T$. This question has been investigated by several authors; see, for example, Browder[1], Halpern[4], Singh and Watson[8], Marino and Trombetta[6], and others. Recently, Xu and Yin[10] proved that if $C$ is a nonempty closed convex subset of a Hilbert space $H$, if $T: C \rightarrow H$ is a nonexpansive nonself-mapping, and if $\left\{x_{t}\right\}$ is the sequence defined by (2) which is bounded, then $\left\{x_{t}\right\}$ converges strongly as $t \rightarrow 1$ to a fixed point of $T$. Next, consider a sunny nonexpansive retraction $P$ from $E$ onto $C$. Then, following Marino and Trombetta[6], for a given $u \in C$ and each $t \in(0,1)$, we define contractions $S_{t}$ and $U_{t}$ from 
$C$ into itself by

$$
S_{t} x=t P T x+(1-t) u \quad \text { for all } x \in C
$$

and

$$
U_{t} x=P(t T x+(1-t) u) \quad \text { for all } x \in C .
$$

By Banach's contraction principle, there exists a unique fixed point $x_{t}$ (resp. $y_{t}$ ) of $S_{t}$ (resp. $\left.U_{t}\right)$ in $C$, i.e.,

$$
x_{t}=t P T x_{t}+(1-t) u
$$

and

$$
y_{t}=P\left(t T y_{t}+(1-t) u\right)
$$

$\mathrm{Xu}$ and Yin[10] also proved that if $C$ is a nonempty closed convex subset of a Hilbert space $H$, if $T: C \rightarrow H$ is a nonexpansive nonself-mapping satisfying the weak inwardness condition, and if $P$ is the nearest projection from $H$ onto $C$, then the sequence $\left\{x_{t}\right\}$ (resp. $\left\{y_{t}\right\}$ ) defined by (3) (resp. (4)) which is bounded converges strongly as $t \rightarrow 1$ to a fixed point of $T$.

In this paper, we extend $\mathrm{Xu}$ and Yin's results[10] to Banach spaces, that is, we prove that the sequence defined by (2)(resp. (3), (4)) which is bounded in a smooth and reflexive Banach space converges strongly as $t \rightarrow 1$ to a fixed point of $T$.

\section{Preliminaries}

Throughout this paper we denote by $E$ and $E^{*}$ a Banach space and the dual space of $E$, respectively. The value of $x^{*} \in E^{*}$ at $x \in E$ will be denoted by $\left\langle x, x^{*}\right\rangle$. We also denote by $F(T)$ the set of all fixed points of $T$, i.e., $F(T)=\{x \in C: T x=x\}$ and by $R$ and $R^{+}$ the sets of all real numbers and all nonnegative real numbers, respectively. When $\left\{x_{n}\right\}$ is a sequence in $E$, then $x_{n} \rightarrow x$ (resp. $x_{n} \rightarrow x, x_{n}^{*} x$ ) will denote strong (resp. weak, weak $k^{*}$ ) convergence of the sequence $\left\{x_{n}\right\}$ to $x$. Let $C$ be a nonempty closed convex subset of $E$, let $D$ be a subset of $C$ and let $P$ be a mapping of $C$ into $D$. Then $P$ is said to be 
sunny if

$$
P(P x+t(x-P x))=P x
$$

whenever $P x+t(x-P x) \in C$ for $x \in C$ and $t \geq 0$. A mapping $P$ of $C$ into $C$ is said to be a retraction if $P^{2}=P$. If a mapping $P$ of $C$ into $C$ is a retraction, then $P z=z$ for every $z \in R(P)$, where $R(P)$ is the range of $P$. A subset $D$ of $C$ is said to be a sunny nonexpansive retract of $C$ if there exists a sunny nonexpansive retraction of $C$ onto $D$; for more details, see[5]. For every $\epsilon$ with $0 \leq \epsilon \leq 2$, the modulus $\delta(\epsilon)$ of convexity of $E$ is defined by

$$
\delta(\epsilon)=\inf \left\{1-\left\|\frac{x+y}{2}\right\|:\|x\| \leq 1, \quad\|y\| \leq 1, \quad\|x-y\| \geq \epsilon\right\}
$$

$E$ is said to be uniformly convex if $\delta(\epsilon)>0$ for every $\epsilon>0$. If $E$ is uniformly convex, then $E$ is reflexive. Let $S(E)=\{x \in E:\|x\|=1\}$. Then the norm of $E$ is said to be Gâteaux differentiable (and $E$ is said to be smooth) if

$$
\lim _{t \rightarrow 0} \frac{\|x+t y\|-\|x\|}{t}
$$

exists for each $x$ and $y$ in $S(E)$. It is also said to be uniformly Fréchet differentiable (and $E$ is said to be uniformly smooth) if the limit (5) is attained uniformly for $x, y$ in $S(E)$. With each $x \in E$, we associate the set

$$
J_{\phi}(x)=\left\{x^{*} \in E^{*} ;\left\langle x, x^{*}\right\rangle=\|x\|\left\|x^{*}\right\| \text { and }\left\|x^{*}\right\|=\phi(\|x\|)\right\}
$$

where $\phi: R^{+} \rightarrow R^{+}$is a continuous and strictly increasing function with $\phi(0)=0$ and $\phi(\infty)=\infty$. Then $J_{\phi}: E \rightarrow 2^{E^{*}}$ is said to be the duality mapping. Suppose that $J_{\phi}$ is single-valued. Then $J_{\phi}$ is said to be weakly sequentially continuous if for each $\left\{x_{n}\right\} \in E$ with $x_{n} \rightarrow x, J_{\phi}\left(x_{n}\right)^{*} J_{\phi}(x)$. For abbreviation, we set $J:=J_{\phi}$. In all our proofs we assume, without loss of generality, that $J$ is normalized. It is well known if $E$ is smooth, then the duality mapping $J$ is single-valued and strong-weak* continuous. It is also known that $E$ is uniformly smooth if and only if $E^{*}$ is uniformly convex; for more details, see 
Diestel[2]. A Banach space $E$ is said to satisfy Opial's condition[7] if for any sequence $\left\{x_{n}\right\}$ in $E, x_{n} \rightarrow x$ implies that

$$
\limsup _{n \rightarrow \infty}\left\|x_{n}-x\right\|<\limsup _{n \rightarrow \infty}\left\|x_{n}-y\right\|
$$

for all $y \in E$ with $y \neq x$. We know that if $E$ admits a weakly sequentially continuous duality mapping, then $E$ satisfies Opial's condition; see[3].

\section{Strong convergence Theorems}

In this section, we first prove a strong convergence theorem for nonexpansive nonselfmappings in a Banach space which generalizes $\mathrm{Xu}$ and Yin's result[10].

Theorem 1 Let $E$ be a smooth and reflexive Banach space with a weakly sequentially continuous duality mapping $J: E \rightarrow E^{*}$, let $C$ be a nonempty closed convex subset of $E$, and let $T: C \rightarrow E$ be a nonexpansive nonself-mapping. Suppose that for some $u \in C$ and each $t \in(0,1)$, the contraction $T_{t}$ defined by (1) has a (unique) fixed point $x_{t} \in C$. Then $T$ has a fixed point if and only if $\left\{x_{t}\right\}$ remains bounded as $t \rightarrow 1$. In this case, $\left\{x_{t}\right\}$ converges strongly as $t \rightarrow 1$ to a fixed point of $T$.

Proof. Let $x$ be a fixed point of $T$. Then we have

$$
\begin{aligned}
\left\|x-x_{t}\right\| & =\left\|x-t T x_{t}-(1-t) u\right\| \\
& \leq t\left\|x-T x_{t}\right\|+(1-t)\|x-u\| \\
& \leq t\left\|x-x_{t}\right\|+(1-t)\|x-u\|
\end{aligned}
$$

and hence $\left\|x-x_{t}\right\| \leq\|x-u\|$. So, $\left\{x_{t}\right\}$ is bounded. Conversely, suppose that $\left\{x_{t}\right\}$ is bounded when $t$ is closed enough to 1 . Then there exist a subsequence $\left\{x_{t_{n}}\right\}$ of the sequence $\left\{x_{t}\right\}$ and a point $y \in C$ such that $x_{t_{n}} \rightarrow y$. By (2), we have

$$
\left\|x_{t_{n}}-T x_{t_{n}}\right\|=\left(1-t_{n}\right)\left\|u-T x_{t_{n}}\right\| \rightarrow 0 \quad \text { as } n \rightarrow \infty .
$$


So, we have

$$
\begin{aligned}
\limsup _{n \rightarrow \infty}\left\|x_{t_{n}}-T y\right\| & \leq \limsup _{n \rightarrow \infty}\left\{\left\|x_{t_{n}}-T x_{t_{n}}\right\|+\left\|T x_{t_{n}}-T y\right\|\right\} \\
& \leq \limsup _{n \rightarrow \infty}\left\|x_{t_{n}}-y\right\| .
\end{aligned}
$$

If $T y \neq y$, from Theorem 1 in [3], we have

$$
\begin{aligned}
\limsup _{n \rightarrow \infty}\left\|x_{t_{n}}-y\right\| & <\limsup _{n \rightarrow \infty}\left\|x_{t_{n}}-T y\right\| \\
& \leq \limsup _{n \rightarrow \infty}\left\|x_{t_{n}}-y\right\| .
\end{aligned}
$$

This is a contradiction. Hence we have $y \in F(T)$. Since, for any $w \in F(T)$,

$$
\begin{aligned}
\left\langle\frac{1}{t_{n}} x_{t_{n}}-\left(\frac{1}{t_{n}}-1\right) u-w, J\left(w-x_{t_{n}}\right)\right\rangle & =\left\langle T x_{t_{n}}-T w, J\left(w-x_{t_{n}}\right)\right\rangle \\
& \geq-\left\|T x_{t_{n}}-T w\right\|\left\|J\left(w-x_{t_{n}}\right)\right\| \\
& \geq-\left\|w-x_{t_{n}}\right\|^{2} \\
& =\left\langle x_{t_{n}}-w, J\left(w-x_{t_{n}}\right)\right\rangle,
\end{aligned}
$$

we have $\left\langle\left(\frac{1}{t_{n}}-1\right)\left(x_{t_{n}}-u\right), J\left(w-x_{t_{n}}\right)\right\rangle \geq 0$. So, we have

$$
\left\langle x_{t_{n}}-u, J\left(w-x_{t_{n}}\right)\right\rangle \geq 0
$$

Thus putting $w=y$,

$$
\begin{aligned}
\left\langle y-u, J\left(y-x_{t_{n}}\right)\right\rangle & =\left\langle y-x_{t_{n}}, J\left(y-x_{t_{n}}\right)\right\rangle+\left\langle x_{t_{n}}-u, J\left(y-x_{t_{n}}\right)\right\rangle \\
& \geq\left\|y-x_{t_{n}}\right\|^{2} .
\end{aligned}
$$

Since $x_{t_{n}} \rightarrow y$ and $J$ is weakly sequentially continuous, we have $x_{t_{n}} \rightarrow y$. By using the argument above again, we obtain a subsequence $\left\{x_{t_{m}}\right\}$ of $\left\{x_{t}\right\}$ converging weakly to some $z \in C$ such that $z=T z$ and $x_{t_{m}} \rightarrow z$. From (6), we have

$$
\langle y-u, J(w-y)\rangle \geq 0 \text { and }\langle z-u, J(w-z)\rangle \geq 0
$$

for any $w \in F(T)$ and hence

$$
\langle y-u, J(z-y)\rangle \geq 0 \text { and }\langle z-u, J(y-z)\rangle \geq 0
$$


This implies $y=z$. Therefore we have $x_{t} \rightarrow z$.

Next, we consider two strong convergence theorems which generalize $\mathrm{Xu}$ and Yin's results[10], using a sunny nonexpansive retraction $P$ from $E$ onto $C$. Let $E$ be a Banach space and let $C$ be a nonempty convex subset of $E$. Then for $x \in C$ we define the inward set $I_{C}(x)$ as follows:

$$
I_{C}(x)=\{y \in E: y=x+a(z-x) \text { for some } z \in C \text { and } a \geq 0\}
$$

A mapping $T: C \rightarrow E$ is said to be inward if $T x \in I_{C}(x)$ for all $x \in C . T$ is also said to be weakly inward if for each $x \in C, T x$ belongs to the closure of $I_{C}(x)$.

Theorem 2 Let $E$ be a smooth and reflexive Banach space with a weakly sequentially continuous duality mapping $J: E \rightarrow E^{*}$, let $C$ be a nonempty closed convex subset of $E$, and let $T: C \rightarrow E$ be a nonexpansive nonself-mapping satisfying the weak inwardness condition. Suppose that $C$ is a sunny nonexpansive retract of $E$ and for some $u \in C$ and each $t \in(0,1), x_{t} \in C$ is a (unique) fixed point of the contraction $S_{t}$ defined by (3), where $P$ is a sunny nonexpansive retraction of $E$ onto $C$. Then $T$ has a fixed point if and only if $\left\{x_{t}\right\}$ remains bounded as $t \rightarrow 1$. In this case, $\left\{x_{t}\right\}$ converges strongly as $t \rightarrow 1$ to a fixed point of $T$.

Proof. Let $x$ be a fixed point of $T$. Then $\left\{x_{t}\right\}$ is bounded. Conversely, suppose that $\left\{x_{t}\right\}$ is bounded when $t$ is closed enough to 1 . Applying Theorem 1, we obtain that $\left\{x_{t}\right\}$ converges strongly as $t \rightarrow 1$ to a fixed point $z$ of $P T$. Next, let us show $z \in F(T)$. Since $z=P T z$ and $P$ is a sunny nonexpansive retraction of $E$ onto $C$, we have

$$
\langle T z-z, J(z-v)\rangle \geq 0
$$

for all $v \in C$; see[9]. On the other hand, $T z$ belongs to the closure of $I_{C}(z)$ by the weak inwardness condition. Hence there exist, for each integer $n \geq 1, z_{n} \in C$ and $a_{n} \geq 0$ such that the sequence

$$
y_{n}:=z+a_{n}\left(z_{n}-z\right) \rightarrow T z .
$$


Since

$$
\begin{aligned}
0 & \leq a_{n}\left\langle T z-z, J\left(z-z_{n}\right)\right\rangle \\
& =\left\langle T z-z, J\left(a_{n}\left(z-z_{n}\right)\right)\right\rangle \\
& =\left\langle T z-z, J\left(z-y_{n}\right)\right\rangle
\end{aligned}
$$

and $J$ is weakly sequentially continuous, we have

$$
0 \leq\langle T z-z, J(z-T z)\rangle=-\|T z-z\|^{2}
$$

and hence $T z=z$.

Theorem 3 Let $E$ be a smooth and reflexive Banach space with a weakly sequentially continuous duality mapping $J: E \rightarrow E^{*}$, let $C$ be a nonempty closed convex subset of $E$, and let $T: C \rightarrow E$ be a nonexpansive nonself-mapping satisfying the weak inwardness condition. Suppose that $C$ is a sunny nonexpansive retract of $E$ and for some $u \in C$ and each $t \in(0,1), y_{t} \in C$ is a (unique) fixed point of the contraction $U_{t}$ defined by (4), where $P$ is a sunny nonexpansive retraction of $E$ onto $C$. Then $T$ has a fixed point if and only if $\left\{y_{t}\right\}$ remains bounded as $t \rightarrow 1$. In this case, $\left\{y_{t}\right\}$ converges strongly as $t \rightarrow 1$ to a fixed point of $T$.

Proof. Let $x$ be a fixed point of $T$. Then we have

$$
\begin{aligned}
\left\|x-y_{t}\right\| & =\left\|P x-P\left(t T y_{t}+(1-t) u\right)\right\| \\
& \leq t\left\|x-T y_{t}\right\|+(1-t)\|x-u\| \\
& \leq t\left\|x-y_{t}\right\|+(1-t)\|x-u\|
\end{aligned}
$$

and hence $\left\|x-y_{t}\right\| \leq\|x-u\|$. So, $\left\{y_{t}\right\}$ is bounded. Conversely, suppose that $\left\{y_{t}\right\}$ is bounded when $t$ is closed enough to 1 . Then there exist a subsequence $\left\{y_{t_{n}}\right\}$ of the sequence $\left\{y_{t}\right\}$ and a point $y \in C$ such that $y_{t_{n}} \rightarrow y$. Since $\left\{T y_{t_{n}}\right\}$ is bounded and

$$
\begin{aligned}
\left\|y_{t_{n}}-P T y_{t_{n}}\right\| & \leq\left\|t_{n} T y_{t_{n}}+\left(1-t_{n}\right) u-T y_{t_{n}}\right\| \\
& =\left(1-t_{n}\right)\left\|u-T y_{t_{n}}\right\|
\end{aligned}
$$


we have $y_{t_{n}}-P T y_{t_{n}} \rightarrow 0$. So, we have

$$
\begin{aligned}
\limsup _{n \rightarrow \infty}\left\|y_{t_{n}}-P T y\right\| & \leq \limsup _{n \rightarrow \infty}\left\{\left\|y_{t_{n}}-P T y_{t_{n}}\right\|+\left\|P T y_{t_{n}}-P T y\right\|\right\} \\
& \leq \limsup _{n \rightarrow \infty}\left\|y_{t_{n}}-y\right\| .
\end{aligned}
$$

If $P T y \neq y$, from Theorem 1 in [3], we have

$$
\begin{aligned}
\limsup _{n \rightarrow \infty}\left\|y_{t_{n}}-y\right\| & <\limsup _{n \rightarrow \infty}\left\|y_{t_{n}}-P T y\right\| \\
& \leq \limsup _{n \rightarrow \infty}\left\|y_{t_{n}}-y\right\| .
\end{aligned}
$$

This is a contradiction. Hence $y=P T y$. So, from [9],

$$
\langle T y-y, J(y-v)\rangle \geq 0
$$

for all $v \in C$. On the other hand, $T y$ belongs to the closure of $I_{C}(y)$ by the weak inwardness condition. Hence there exist, for each integer $n \geq 1, z_{n} \in C$ and $a_{n} \geq 0$ such that the sequence

$$
y_{n}:=y+a_{n}\left(z_{n}-y\right) \rightarrow T y .
$$

As in the proof of Theorem 2, we have $T y=y$. For any $w \in F(T)$, we have

$$
t(w-u)+u=t w+(1-t) u=P(t w+(1-t) u)
$$

and hence

$$
\begin{aligned}
\left\|\left(y_{t}-u\right)-t(w-u)\right\|^{2} & =\left\|P\left(t T y_{t}+(1-t) u\right)-u-t(w-u)\right\|^{2} \\
& =\left\|P\left(t\left(T y_{t}-u\right)+u\right)-u-t(w-u)\right\|^{2} \\
& =\left\|P\left(t\left(T y_{t}-u\right)+u\right)-u-P(t(w-u)+u)+u\right\|^{2} \\
& \leq\left\|t\left(T y_{t}-u\right)-t(w-u)\right\|^{2} \\
& \leq t^{2}\left\|y_{t}-w\right\|^{2} \\
& =t^{2}\left\|\left(y_{t}-u\right)-(w-u)\right\|^{2} .
\end{aligned}
$$


So, we have

$$
\begin{aligned}
0 & \geq\left\|\left(y_{t}-u\right)-t(w-u)\right\|^{2}-\left\|t\left(y_{t}-u\right)-t(w-u)\right\|^{2} \\
& \geq 2\left\langle(1-t)\left(y_{t}-u\right), J\left(t\left(y_{t}-w\right)\right)\right\rangle \\
& =2(1-t) t\left\langle y_{t}-u, J\left(y_{t}-w\right)\right\rangle
\end{aligned}
$$

and hence

$$
\left\langle y_{t}-u, J\left(y_{t}-w\right)\right\rangle \leq 0
$$

Thus putting $w=y$,

$$
\begin{aligned}
\left\langle y-u, J\left(y-y_{t_{n}}\right)\right\rangle & =\left\langle y-y_{t_{n}}, J\left(y-y_{t_{n}}\right)\right\rangle+\left\langle y_{t_{n}}-u, J\left(y-y_{t_{n}}\right)\right\rangle \\
& \geq\left\|y-y_{t_{n}}\right\|^{2} .
\end{aligned}
$$

Since $y_{t_{n}}-y$ and $J$ is weakly sequentially continuous, we have $y_{t_{n}} \rightarrow y$. As in the proof of Theorem 1 , we have $y_{t} \rightarrow z$.

\section{References}

[1] F.E. Browder, Convergence of approximants to fixed points of nonexpansive nonlinear mappings in Banach spaces, Archs. Ration. Mech. Anal., 24 (1967), 82-90.

[2] J. Diestel, Geometry of Banach spaces-selected topics, Lecture Notes in Math., Vol. 485, Springer-Verlag, Berlin, Heidelberg, and New York, 1975.

[3] J.P. Gossez and E.L. Dozo, Some geometric properties related to the fixed point theory for nonexpansive mappings, Pacific J. Math., 40(3) (1972), 565-573.

[4] B. Halpern, Fixed points of nonexpanding maps, Bull. Am. Math. Soc., 73 (1967), 957-961.

[5] S. Kitahara and W. Takahashi, Image recovery by convex combinations of sunny nonexpansive Retractions, Topol. Methods Nonlinear Anal., 2 (1993), 333-342. 
[6] G. Marino and G. Trombetta, On approximating fixed points for nonexpansive maps, Indian J. Math., 34 (1992), 91-98.

[7] Z. Opial, Weak convergence of the sequence of successive approximations for nonexpansive mappings, Bull. Amer. Math. Soc., 73 (1967), 591-597.

[8] S.P. Singh and B. Watson, On approximating fixed points, Proc. Symp. Pure Math., 45 (1986), 393-395.

[9] W. Takahashi, Nonlinear Functional Analysis (Japanese), Kindaikagaku, Tokyo, 1988.

[10] H.K. Xu and X.M. Yin, Strong convergence theorems for nonexpansive nonselfmappings, Nonlinear Analysis., 24 (1995), 223-228.

(G.E. Kim) DEPARTMENT OF INFORMATION SCIENCES, TOKYO INSTITUTE OF TECHNOLOGY, OHOKAYAMA, MEGURO-KU, TOKYO 152, JAPAN

E-mail address, G.E. Kim: kim@is.titech.ac.jp

(W. Takahashi) DEPARTMENT OF INFORMATION SCIENCES, TOKYO INSTITUTE OF TECHNOLOGY, OHOKAYAMA, MEGURO-KU, TOKYO 152, JAPAN

E-mail address, W. Takahashi: wataru@is.titech.ac.jp

Received December 28, 1995 